\title{
A!
}

This is an electronic reprint of the original article.

This reprint may differ from the original in pagination and typographic detail.

Mikheev, Konstantin G.; Saushin, Aleksandr S.; Zonov, Ruslan G.; Nasibulin, Albert G.; Mikheev, Gennady M.

Photon-drag in single-walled carbon nanotube and silver-palladium films

Published in:

Journal of Nanophotonics

DOI:

10.1117/1.JNP.10.012505

Published: 31/03/2016

Document Version

Publisher's PDF, also known as Version of record

Please cite the original version:

Mikheev, K. G., Saushin, A. S., Zonov, R. G., Nasibulin, A. G., \& Mikheev, G. M. (2016). Photon-drag in singlewalled carbon nanotube and silver-palladium films: The effect of polarization. Journal of Nanophotonics, 10(1), 1-9. [012505]. https://doi.org/10.1117/1.JNP.10.012505

This material is protected by copyright and other intellectual property rights, and duplication or sale of all or part of any of the repository collections is not permitted, except that material may be duplicated by you for your research use or educational purposes in electronic or print form. You must obtain permission for any other use. Electronic or print copies may not be offered, whether for sale or otherwise to anyone who is not an authorised user. 


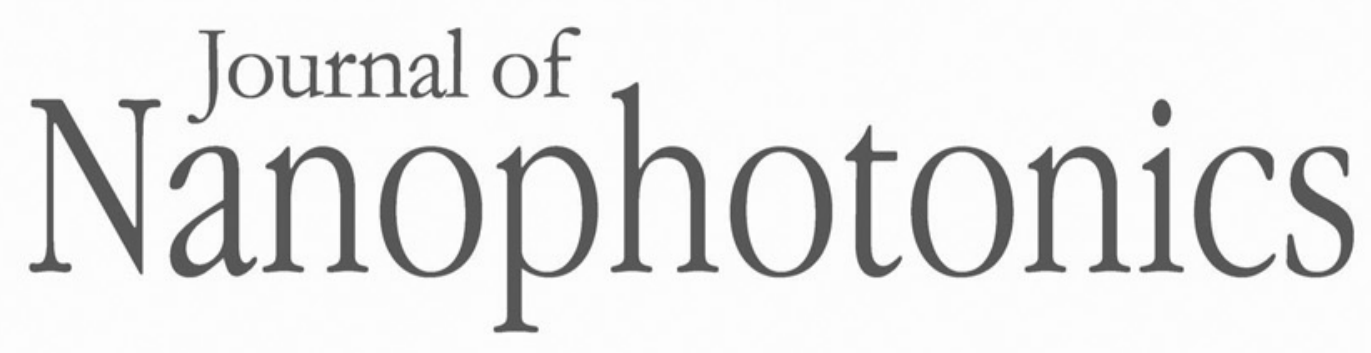

Nanophotonics.SPIEDigitalLibrary.org

\section{Photon-drag in single-walled carbon nanotube and silver-palladium films: the effect of polarization}

Konstantin G. Mikheev

Aleksandr S. Saushin

Ruslan G. Zonov

Albert G. Nasibulin

Gennady M. Mikheev 


\title{
Photon-drag in single-walled carbon nanotube and silver-palladium films: the effect of polarization
}

\author{
Konstantin G. Mikheev, ${ }^{\text {a }}$ 网 Aleksandr S. Saushin, ${ }^{\mathrm{a}}$ Ruslan G. Zonov, ${ }^{\mathrm{a}}$ \\ Albert G. Nasibulin, ${ }^{\mathrm{b}, \mathrm{c}, \mathrm{d}}$ and Gennady M. Mikheev ${ }^{\mathrm{a}}$ \\ ${ }^{a}$ Institute of Mechanics Ural Branch of Russian Academy of Sciences, ul. T. Baramzinoy 34, \\ Izhevsk 426067, Russia \\ ${ }^{\mathrm{b}}$ Aalto University, Department of Applied Physics, P.O. Box 11100, Espoo, \\ FI-00076 AALTO, Finland \\ ${ }^{\mathrm{c}}$ St. Petersburg Polytechnic University, 29, Polytechnicheskaya Street, \\ St. Petersburg 195251, Russia \\ ${ }^{\mathrm{d} S}$ Skolkovo Institute of Science and Technology, Skolkovo, Nobel Street 3, \\ Moscow 143026, Russia
}

\begin{abstract}
Polarization influence on the photovoltaic current raised due to the photon-drag effect in the single-walled carbon nanotube (SWNT) films and nanostructured silver-palladium (Ag/ Pd) resistive films is examined at the wavelengths of 532 and $1064 \mathrm{~nm}$ of nanosecond laser pulses. The SWNT films were synthesized by the aerosol chemical vapor deposition technique. $\mathrm{Ag} / \mathrm{Pd}$ films, consisting of $\mathrm{AgPd}$ alloy and palladium oxide ( $\mathrm{PdO})$, were prepared by burning a special paste on a ceramic substrate. The films obtained were characterized by Raman spectroscopy. It is shown that the $\mathrm{Ag} / \mathrm{Pd}$ films Raman spectra consist of $\mathrm{PdO}$ peak that moves from $650 \mathrm{~cm}^{-1}$ to $628 \mathrm{~cm}^{-1}$ as the excitation He-Ne laser power increases. The photocurrent was measured at the oblique incidence of the laser beam on the film in the direction perpendicular to the plane of incidence. It is found that the transverse photocurrent in the SWNT films at circular polarization is absent and does not depend on the direction of the electric field vector rotation (the sign of the circular polarization) of the incident irradiation. The photocurrent in the $\mathrm{Ag} / \mathrm{Pd}$ films at circular polarized irradiation is significant and depends on the circular polarization sign. The results obtained demonstrate the potential applications of the $\mathrm{Ag} / \mathrm{Pd}$ resistive films as a sensor of the circular polarization sign of the incident light pulse in a wide wavelength range. () 2015 Society of Photo-Optical Instrumentation Engineers (SPIE) [DOI: 10.1117/1.JNP10.012505]
\end{abstract}

Keywords: photon-drag effect; single-walled carbon nanotube film; silver-palladium resistive film; Raman spectroscopy; laser pulse; transverse photocurrent; photocurrent; circular polarization sign.

Paper 15073SSP received Aug. 3, 2015; accepted for publication Sep. 15, 2015; published online Nov. 5, 2015.

\section{Introduction}

The photovoltaic current generation, depending on the direction of the wave vector and the polarization of incident laser radiation, is observed in various nanocarbon film materials [nanographite films, graphene, and single-walled carbon nanotube (SWNT) films]. ‥ phenomena have been observed in quantum wells, 4 gold films of nanometer thickness with different morphologies, 明 and also in nanostructured $\mathrm{Ag} / \mathrm{Pd}$ resistive films. 4 The photovoltaic current dependence on the direction of the wave vector in centrosymmetrical media can be explained by the photon-drag effect, which is observed both at linear and circular polarizations. . The study of polarization influence on the photon-drag effect in different film structures is of interest in terms of developing and creating the polarization analyzers, operating on new principles. 9 四

*Address all correspondence to: Konstantin G. Mikheev, E-mail: 耳mikheev@udmanru

1934-2608/2015/\$25.00 (C) 2015 SPIE 
The aim of this paper is to carry out the comparative study of a photovoltaic signal due to the photon-drag effect in SWNT films and Ag/Pd films as a function of the polarization ellipticity and the rotation direction of the electrical field vector of the incident laser radiation.

\section{Materials}

\subsection{Brief Description of the Production Technology of the Single-Walled Carbon Nanotube Films}

SWNT film is a new material with a number of unique properties. They are widely used in photonics and optoelectronics. There are a large number of papers showing the possibility of using the SWNT films for passive Q-switching of lasers in order to obtain picosecond and femtosecond laser pulses in a wide spectral range (see review 매는). The sensors of bolometric

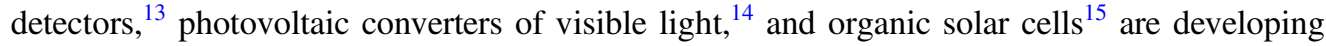
based on the SWNT films.

SWNTs were synthesized by an aerosol (floating catalyst) method based on thermal decomposition of ferrocene vapor in a carbon monoxide (CO) atmosphere ${ }^{-1}$ The synthesis was carried in a scaled-up version of the reactor with a tube of $150 \mathrm{~mm}$ in diameter and $1.5 \mathrm{~m}$ in length at a total $\mathrm{CO}$ flow rate of $4 \mathrm{~L} / \mathrm{min}$ (with $1 \%$ volumetric content of $\mathrm{CO}_{2}$ ) and at a temperature of $880^{\circ} \mathrm{C} \square$ Ferrocene was evaporated at room temperature and thermally decomposed in a high temperature gradient resulting in the supersaturated conditions, formation of nanoparticles, and subsequent CO decomposition on the surface of catalyst particles leading to the SWNT growth. The synthesized SWNTs were collected downstream of the reactor by passing the aerosol flow through nitrocellulose membrane filters to form SWNT films, which were transferred from the low adhesive force filter to a polyethylene terephthalate (PET) substrate by a simple room temperature press transfer process $\$$ The SWNT collection time was varied from 3 to $30 \mathrm{~min}$, which corresponded to the SWNT film thickness change from 25 to $625 \mathrm{~nm}$ and film transmittance from $94 \%$ to $22 \%$.

\subsection{Brief Description of the Production Technology of Ag/Pd Films}

$\mathrm{Ag} / \mathrm{Pd}$ resistive films have stable electrical characteristics and have long been used in electronics. ${ }^{\square}$ They are widely applied for obtaining hybrid microcircuits, multicrystal modules, integrated circuits assemblies, and passive electronic components such as resistors, inductive elements, and multilayer capacitors. ${ }^{20}$ Recently, it was shown that $\mathrm{Ag} / \mathrm{Pd}$ resistive films can be used as photovoltaic converters which are sensitive to the direction of the incident radiation wave vector. It is established that they can operate as such convertors in a wide spectral range from 266 to $1064 \mathrm{~nm}$.?

The Ag/Pd films are produced using technologies ${ }^{22}$ based on burning a special paste which contains silver oxide and palladium on the insulating substrate surface at high temperatures. The applied paste layer is dried and fired, i.e., is subjected to heat treatment, which imparts certain electrical and mechanical properties to the film. The paste includes functional, structural, and technological components. The functional component is the principal one determining the basic properties of the obtained films and consists of silver oxide $\left(\operatorname{Ag}_{2} \mathrm{O}\right)$ and palladium. The structural component represents finely divided particles of glass, e.g., STs273 glass, whose melting temperature is lower than the burning temperature. During burning, molten glass wets functional component particles with the suspension formation. After cooling and hardening, a mechanically strong film is formed, within which the quasihomogeneous distribution of functional phase particles takes place. The technological component is a "binder" which imparts certain viscosity and plasticity to the paste. This component contains organic substances (e.g., lanolin, colophony) and a certain solvent. The latter is evaporated during drying, the organic materials decompose and burn down during burning, i.e., the technological component is removed. In the experiments, the $\mathrm{Ag} / \mathrm{Pd}$ films obtained at different fusion temperatures $(878,1013$, and $1113 \mathrm{~K})$ were studied. Finished film obtained at a fusion temperature of $878 \mathrm{~K}$ is a porous material, with the pore radius being of from 25 to $500 \mathrm{~nm}$ [see Fig. 1(a)]..$^{3.24}$ As seen in Figs. 1(b) and [1(c), the film obtained at a 


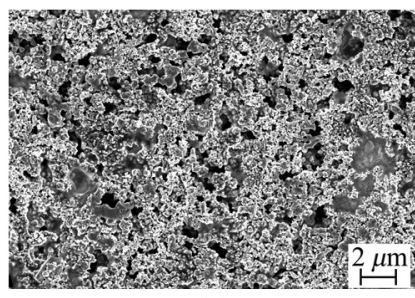

(a)

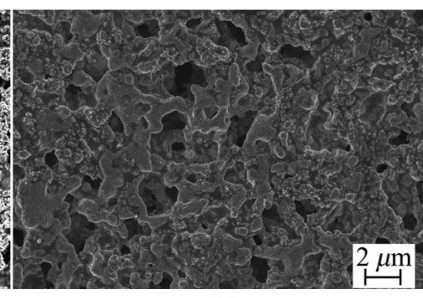

(b)

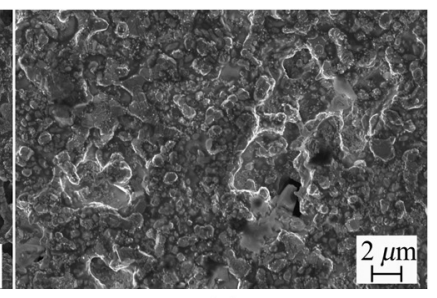

(c)

Fig. 1 Scanning electron microscope images of the resistive $\mathrm{Ag} / \mathrm{Pd}$ films' surface obtained at different fusion temperatures: (a) 878, (b) 1013, and (c) $1113 \mathrm{~K}$.

fusion temperature of $1013 \mathrm{~K}$ is less porous, and the film obtained at a fusion temperature of $1113 \mathrm{~K}$ is not porous. The film's size was $20 \times 20 \mathrm{~mm}^{2}$ and the thickness was $20 \mu \mathrm{m}$.

\subsection{Characterization of the Films}

Raman spectroscopy was used to characterize the SWNT and Ag/Pd films. It was carried out on the Raman spectrometer Labram HR800 using excitation at $632.8 \mathrm{~nm}$, with the $\times 100$ objective used. The maximal excitation power was $8 \mathrm{~mW}$. In order to decrease this, the imbedded neutral filters were used. The Raman spectra of the SWNT and Ag/Pd films were obtained in the range of wavenumbers from 100 to $1700 \mathrm{~cm}^{-1}$ and from 200 to $800 \mathrm{~cm}^{-1}$, respectively. Absorbance spectrum of the SWNT films was measured using double-beam UV/VIS spectrometer PerkinElmer LAMBDA 650.

\subsubsection{Single-Walled Carbon Nanotube Films}

The absorbance spectrum measured from the SWNT film transferred on a PET substrate is presented in Fig. 国 The spectrum was obtained against an empty PET film. The Van Hove transition (at the wavelength range from 700 to $800 \mathrm{~nm}$ ) of metallic SWNTs is clearly visible, indicating the high quality of the SWNTs' sample. 1

Raman spectrum measured from the SWNT film on the PET substrate at a low laser power density of $0.4 \mathrm{~kW} / \mathrm{cm}^{2}$ (to avoid laser heating and modification of the SWNT film 2 [26) shows the strong $\mathrm{G}$ band [Fig. B(a)] and radial breathing modes (RBMs) [Fig. 3(b)]. The $\mathrm{G}$ band is related to the vibration of $\mathrm{sp}^{2}$-bonded carbon atoms in a two-dimensional hexagonal lattice of the graphite layer, while the $\mathrm{D}$ band corresponds to the presence of defects on the nanotube walls or amorphous carbon material; therefore, the intensity ratio of $\mathrm{G}$ and D bands $\left(I_{G} / I_{D}\right)$ is used as a reflection of the SWNT samples' quality. ${ }^{2}$ The $I_{G} / I_{D}$ ratio of the SWNT film on the PET substrate is equal to 36 , which shows the good quality of the SWNTs.

The RBMs correspond to the coherent vibration of the carbon atoms in the radial direction and are used to probe the nanotube diameter $\left(d_{t}\right)$ through its frequency $\left(\omega_{\mathrm{RBM}}\right)$ based on the

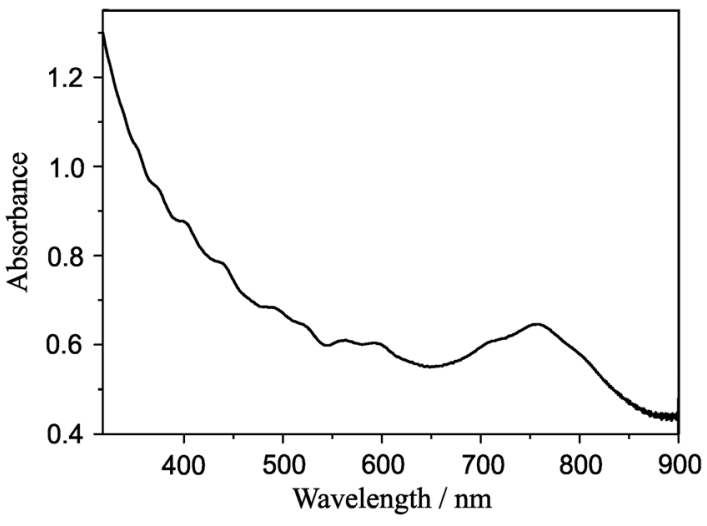

Fig. 2 Absorbance spectrum measured from the single-walled carbon nanotube (SWNT) films on the polyethylene terephthalate substrate (PET). 

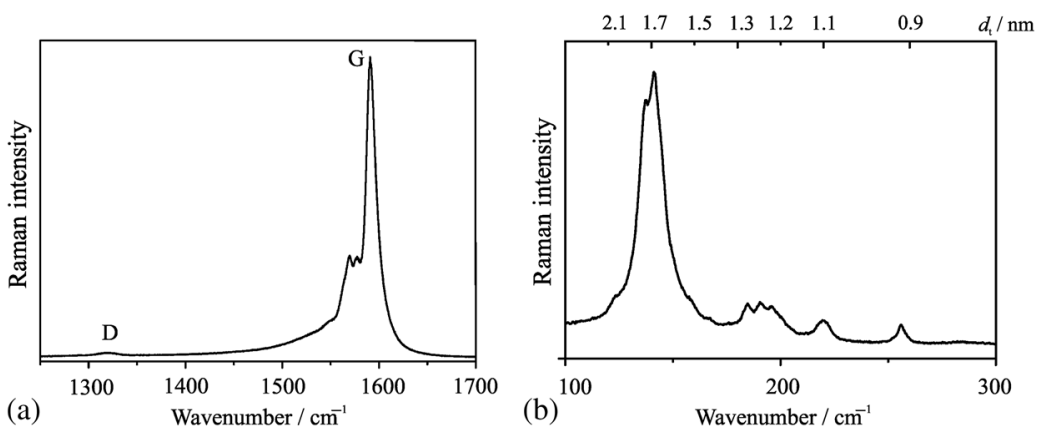

Fig. 3 Raman spectrum of the SWNT film on the PET substrate excited by $632.8 \mathrm{~nm}$ : (a) G and D bands and (b) RBMs. Excitation power density was $0.4 \mathrm{~kW} / \mathrm{cm}^{2}$.

well-established relationship of $\omega_{\mathrm{RBM}}=217.8 / d_{t}+15.7$. ${ }^{\mathrm{g}}$ When excited, the RBM peaks were mainly located in the range of 130 to $160 \mathrm{~cm}^{-1}$, that corresponds to a diameter range of $2.1 \div$ $1.5 \mathrm{~nm}$ (see the upper $\mathrm{x}$-axis) [Fig. B(b)].

\subsection{2 $\mathrm{Ag} / \mathrm{Pd}$ Resistive Films}

In Fig. , the Raman spectra of $\mathrm{Ag} / \mathrm{Pd}$ films obtained from the same resistive pastes at different fusion temperatures are presented. One can see the strong sharp peak in the spectrum of the film obtained at the fusion temperature of $878 \mathrm{~K}$. There is the same peak in the spectrum of the film obtained at the fusion temperature of $1013 \mathrm{~K}$, but it is smaller and there is no such band in the spectrum of the film obtained at the fusion temperature of $1113 \mathrm{~K}$. According to the literature data, ${ }^{0}$ @ this peak with a shift of $649 \mathrm{~cm}^{-1}$ is associated with the $\mathrm{PdO}$ content in the film, so it is clear that the PdO content in the films decreases as the fusion temperature increases. We have established earlier ${ }^{2}$ that the photosensitivity of the $\mathrm{Ag} / \mathrm{Pd}$ films is directly connected with the fusion temperature, e.g., the films obtained at the fusion temperature of $878 \mathrm{~K}$ have the maximal photon-drag current, whereas the films obtained at the fusion temperature of $1113 \mathrm{~K}$ do not possess this photovoltaic phenomenon. Thus, we can conclude that the photosensitivity of the $\mathrm{Ag} / \mathrm{Pd}$ films is directly connected with the $\mathrm{PdO}$ content in them. These results agree with the x-ray structural analysis confirming the effect of the percentage content of the PdO on the photosensitivity of the $\mathrm{Ag} / \mathrm{Pd}$ resistive films. ${ }^{2}$

In Figs. $5(\mathrm{a})$ and $5(\mathrm{~b})$, the Raman spectra of $\mathrm{Ag} / \mathrm{Pd}$ resistive films produced at the fusion temperatures of 878 and $1013 \mathrm{~K}$ obtained at different excitation powers are presented. One can see that the PdO peak wavenumber shifts down and the PdO peak FWHM increases as

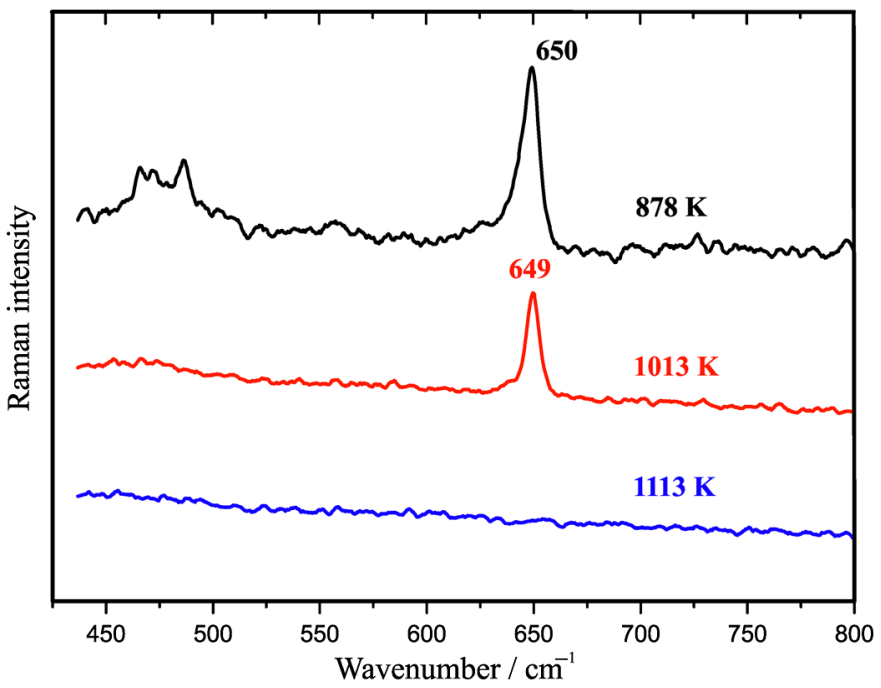

Fig. 4 Raman spectra of $\mathrm{Ag} / \mathrm{Pd}$ films obtained from resistive paste of the same composition at different fusion temperatures excited by $632.8 \mathrm{~nm}$. Excitation power was $0.1 \mathrm{~mW}$. 

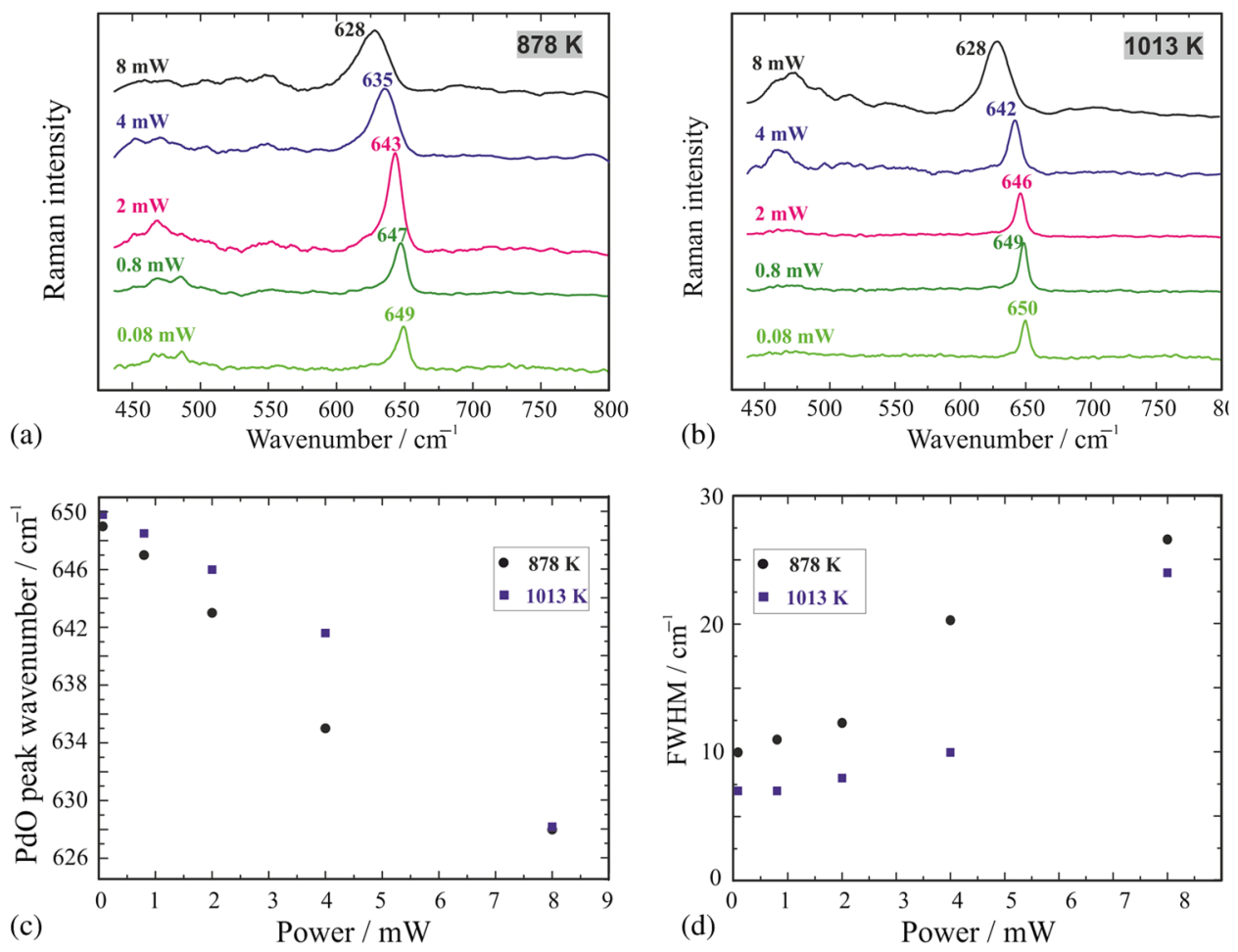

Fig. 5 Raman spectra of Ag/Pd films produced at the fusion temperatures: (a) 878 and (b) $1013 \mathrm{~K}$ measured at different excitation power. (c) $\mathrm{PdO}$ peak wavenumber as a function of excitation power and (d) PdO peak FWHM as a function of excitation power.

the excitation power increases for both of the films [see Figs. 5(c)] and 5(d)]. It is worth noting that this process is reversible, i.e., while the laser power decreases the $\mathrm{PdO}$ peak wavenumber shifts up and the PdO peak FWHM decreases up to the initial points. This suggests that PdO is affected by laser heating but is not destroyed.

\section{Experimental Results}

To study the photovoltaic properties of SWNT and Ag/Pd resistive films, each sample was provided with two parallel silver film electrodes which were arranged on the opposite sides of the square films (see Fig. 6). The photovoltaic properties of the films were studied using a laser generating 16 and $19 \mathrm{~ns}$ pulses at wavelengths of 532 and $1064 \mathrm{~nm}$, respectively. The scheme of the experiments is presented in Fig. 6. The linearly polarized laser pulses were directed

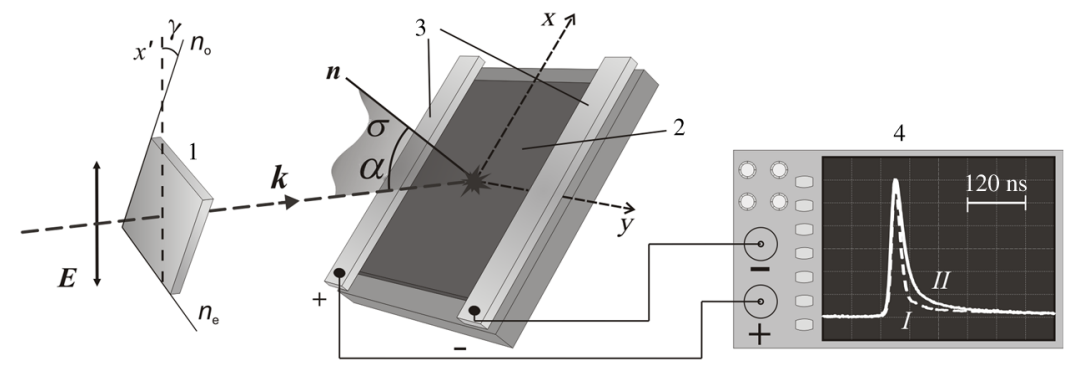

Fig. 6 The scheme of the experiment: (1) quarter-wave plate, (2) the film; (3) electrodes, (4) oscilloscope; $(\boldsymbol{n})$ normal to the film surface, $(\boldsymbol{k})$ wave vector of incident radiation, $(\sigma)$ plane of incidence, $(\alpha)$ angle of incidence, $\left(n_{e}\right)$ optical axis of the quarter-wave plate $\left(n_{e} \perp n_{o}\right)$, $\left(x^{\prime}\right)$ vertical axis, and $(\boldsymbol{E})$ electric field vector $\left(\boldsymbol{E}, \boldsymbol{x}^{\prime} \| \sigma\right)$. The electrodes are parallel to the incidence plane $\sigma$. The pulse shape in the inset is shown for Ag/Pd films: (I) incident laser pulse shape and (II) shape of photovoltaic pulses appearing between electrodes. 
through quarter-wave plate 1 on the films 2 . The angle of radiation incidence on the film was $45 \mathrm{deg}$; the measuring electrodes 3 were parallel to the incidence plane $\sigma$. The linearly polarized laser radiation at the wavelengths of 532 or $1064 \mathrm{~nm}$ was converted into circularly polarized radiation by the proper quarter-wave plate. The angle $\gamma$ of rotation of the quarter-wave plate set a light ellipticity and the sign of circular polarization, where $\gamma$ is the angle between vertical axis $x$, and axis $n_{o}$ perpendicular to the optical axis $n_{e}$ of the quarter-wave plate, see Fig. 6. The photovoltaic signal (pulsed voltage amplitude) appearing between two electrodes upon exposure to light pulses was recorded using a broadband digital oscilloscope 4 (Tektronix TDS7704B) with an input resistance of $50 \Omega$. The electric voltage pulse $U_{y}$ was characterized by the duration and voltage defined as $U_{y}=g A$, where $g$ is the polarity $(g=+1$ or -1$)$ and $A$ is the amplitude $(A \geq 0)$. The typical shape of photovoltaic pulses is shown in Fig. 6 (inset). $\mathrm{Ag} / \mathrm{Pd}$ films obtained at fusion temperatures of 1013 and $1113 \mathrm{~K}$ have not possessed photovoltaic properties due to the low $\mathrm{PdO}$ content, so we would discuss further the properties only of the $\mathrm{Ag} / \mathrm{Pd}$ film obtained at a fusion temperature of $878 \mathrm{~K}$. The photocurrent, which is due to the photon-drag effect, in the SWNT films nonmonotonically depends on the film thickness and has a maximum value at around 300-350 nm, 目 so the experiments were carried out using films with a thickness of $330 \mathrm{~nm}$.

In view of the fact that $U_{y}$ is linearly dependent on incident power $P$ and independent of the irradiated area (at fixed $P$ ), it is possible to construct plots of the radiation power conversion factors $\eta_{\lambda}=U_{y} / P$ versus rotation angle $\gamma$ for wavelengths of 532 and $1064 \mathrm{~nm}$.

The experimental dependences of $\eta_{\lambda}$ as a function of $\gamma$ are presented in Fig. 7 . As can be seen from Fig. 7, both for the SWNT films and Ag/Pd films, the maximum absolute value $\left|\eta_{\lambda}^{\max }\right|$ of factor $\eta_{\lambda}$ decreases with increasing frequency (decreasing wavelength $\lambda$ ) of the incident radiation. For the both type of films at $\gamma=0,90$, and $180 \mathrm{deg}$ ( $p$-polarization), the response signal is absent (additional measurements showed that this signal is also absent for the $s$-polarized radiation). Moreover, the signal at circular polarization for SWNT films is equal to zero. For Ag/Pd films irradiated by radiation at the wavelengths of $\lambda=1064 \mathrm{~nm}$ or $\lambda=532 \mathrm{~nm}$, the signal is positive for the right-hand (clockwise) polarization and negative for the left-hand (anticlockwise) polarization [Fig. 7(a)]. Thus, there is a direct effect of the circular polarization sign on the signal polarity. On the experimental dependences obtained for SWNT films [Fig. 7(b)] with both rightand left-hand polarized light and the phase angle varied within $0<\gamma<90 \mathrm{deg}$ and $90 \mathrm{deg}$ $<\gamma<180 \mathrm{deg}$, respectively, the signal polarity takes both negative and positive values. To within the experimental error, all four experimental dependences presented in Fig. 7 obey the following relation: $\eta_{\lambda}(90 \mathrm{deg}-\gamma)=-\eta_{\lambda}(90 \mathrm{deg}+\gamma)$, where $0<\gamma<90 \mathrm{deg}$, so that functions $\eta(\gamma)$ are antisymmetric relative to axis $\gamma=90 \mathrm{deg}$.
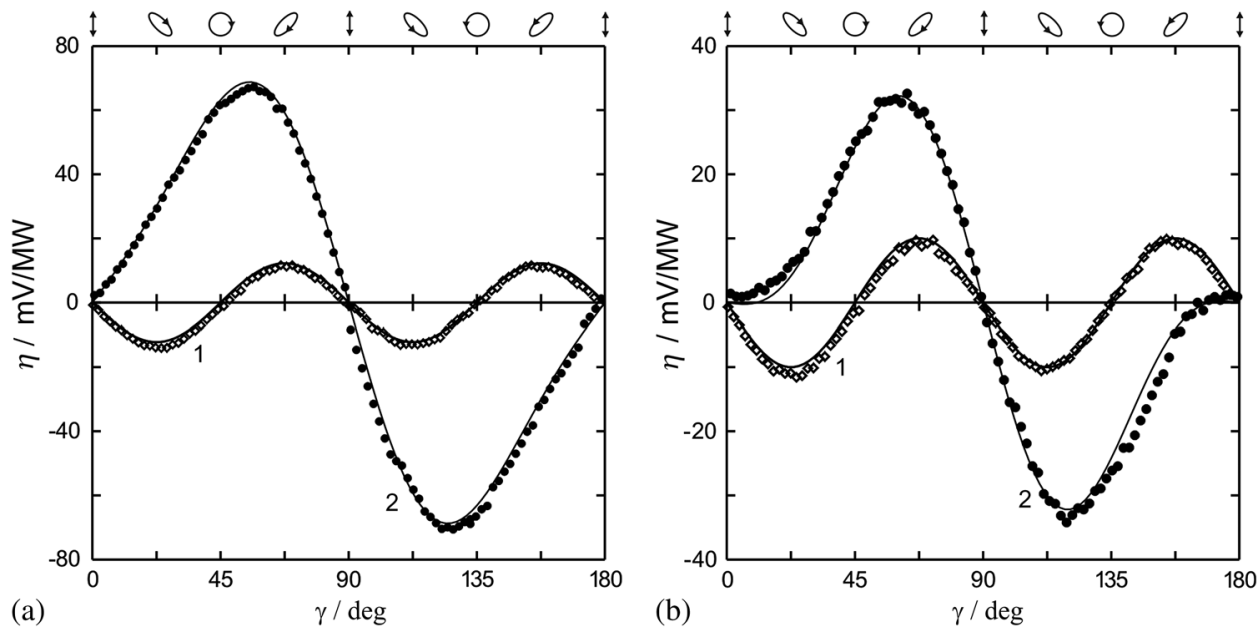

Fig. 7 Plots of conversion coefficient $\eta(\gamma)$ of pulsed laser radiation power into pulsed electric voltage versus phase angle $\gamma$ of a quarter-wave plate for (a) $\lambda=1064 \mathrm{~nm}$, (b) $\lambda=532 \mathrm{~nm}$, for (1) SWNT and (2) Ag/Pd films; points represent experimental data, curves show the approximating functions $\eta_{\lambda}=\eta_{\lambda}^{(1)} \sin (2 \gamma)-\eta_{\lambda}^{(2)} \sin (4 \gamma)$. The top insets show polarization ellipses corresponding to various phase angles $\gamma$. 
All experimental dependences presented in Fig. 7 are approximated by the following function:

$$
\eta_{\lambda}=\eta_{\lambda}^{(1)} \sin (2 \gamma)-\eta_{\lambda}^{(2)} \sin (4 \gamma)
$$

where $\eta_{\lambda}^{(1)}$ and $\eta_{\lambda}^{(2)}$ are the positive factors characterizing the circular and linear contributions ${ }^{2}$ that depend on the radiation wavelength. We found conversion factor $\eta_{\lambda}$ both for SWNT and for Ag/Pd films at two wavelengths. For $\mathrm{Ag} / \mathrm{Pd}$ films at $\lambda=532 \mathrm{~nm}$, the conversion factors are equal to $\eta_{\lambda=532}^{(1)}=24.2$ and $\eta_{\lambda=532}^{(2)}=13 \mathrm{mV} / \mathrm{MW}$, while for the SWNT films $\eta_{\lambda=532}^{(1)}=0, \eta_{\lambda=532}^{(2)}=$ $10 \mathrm{mV} / \mathrm{MW}$. When the radiation wavelength is enhanced to $1064 \mathrm{~nm}$, the circular contribution in $\mathrm{Ag} / \mathrm{Pd}$ films significantly increases in comparison with the linear contribution that considerably changes the form of the dependence; in this case, the factors are equal to $\eta_{\lambda=1064}^{(1)}=63.2$ and $\eta_{\lambda=1064}^{(2)}=14.6 \mathrm{mV} / \mathrm{MW}$. But in nanotube films, the signal insignificantly increases and the factors are equal to $\eta_{\lambda=1064}^{(1)}=0, \eta_{\lambda=1064}^{(2)}=12.2 \mathrm{mV} / \mathrm{MW}$. In other words, at both wavelengths in the nanotube films, the circular contribution is absent and the signal amplitude and polarity are defined by the orientation and the ratio of polarization ellipse axes. The similar results were obtained for the nanographite films. 9 This means that in contrast to the Ag/Pd-films, the SWNT films do not possess the sensitivity to the sign of circular polarization.

The results obtained for the nanographite and the SWNT films are in agreement with those obtained for the photon-drag effect study in graphene in the terahertz wavelength range. ${ }^{\mathbb{T}}$ The circular photon-drag effect in the terahertz range is interpreted as an ac Hall effect. According to Ref. 国 the proportional to $\sin 2 \gamma$ circular contribution of the photon-drag current $i_{y}$ in the direction of $y$, perpendicular to the incidence plane (see Fig. (6) in graphene, disappears at high frequencies of the incident radiation, which is confirmed by our experiments. However, the circular contribution of the drag current in $\mathrm{Ag} / \mathrm{Pd}$ resistive films is significant in the visible and near-infrared regions. According to Ref. 23, Ag/Pd resistive films represent a porous structure of $\mathrm{PdO}$ and a solid solution of $\mathrm{AgPd}$, consisting mainly of $\mathrm{Ag}$, therefore, they have a complex energy structure. Thus, the theoretical description of the circular photon-drag effect in these films is a very difficult task, which is the subject of further research. Nevertheless, we believe that it is already possible to create sensors of the sign of circular polarization of pulsed laser radiation on the basis of resistive $\mathrm{Ag} / \mathrm{Pd}$ films. According to our recent research, such a sensor can operate in the spectral range from 529 to $2940 \mathrm{~nm}$. 33 进

\section{Conclusion}

The photocurrent of the photon-drag effect in the SWNT films in the direction perpendicular to the laser radiation incident plane depends on the orientation and ellipticity of the polarization ellipse of incident laser radiation, does not depend on the rotation direction of the electrical field vector, and vanishes at $p-, s$ - polarizations as well as at the circular polarization of the incident radiation. With the same experimental geometry, the photocurrent of the photon-drag effect in the $\mathrm{Ag} / \mathrm{Pd}$ films, consisting mainly of the AgPd alloy and PdO, vanishes at $p$-, $s$ - polarizations, and depends on the ellipticity and the sign of the laser radiation circular polarization at the wavelengths of 532 and $1064 \mathrm{~nm}$. The results obtained show the opportunity of applying the $\mathrm{Ag} / \mathrm{Pd}$ films for the creation of the laser radiation circular polarization sign sensors operating in a wide spectral range.

\section{Acknowledgments}

This work was supported by the RFBR grants (Project No. 13-08-01031 and Project No. 13-0296037 r_ural_a).

\section{References}

1. G. M. Mikheev et al., "Effect of laser light polarisation on the dc photovoltage response of nanographite films," Quantum Electron. 40(5), 425-430 (2010). 
2. J. Karch et al., "Dynamic hall effect driven by circularly polarized light in a graphene layer," Phys. Rev. Lett. 105(22), 227402 (2010).

3. G. M. Mikheev et al., "Photon-drag effect in single-walled carbon nanotube films," Nang Lett. 12(1), 77-83 (2012).

4. V. A. Shalygin et al., "Spin photocurrents and circular photon drag effect in (110)-grown quantum well structures," DETP Lett. 84(10), 666-672 (2006).

5. T. Hatano et al., "Transverse photovoltage induced by circularly polarized light," Phys. Rev. Lett. 103(10), 103906 (2009).

6. M. Akbari, M. Onoda, and T. Ishihara, "Photo-induced voltage in nano-porous gold thin film," Opt. Express 23(2), 823 (2015).

7. G. M. Mikheev, V. A. Aleksandrov, and A. S. Saushin, "Circular photogalvanic effect observed in silver-palladium film resistors," Tech. Phys. Lett. 37(6), 551-555 (2011).

8. M. M. Glazov and S. D. Ganichev, "High frequency electric field induced nonlinear effects in graphene," Phys. Rep. 535(3), 101-138 (2014).

9. G. M. Mikheev and V. M. Styapshin, "Nanographite analyzer of laser polarization," Instrum. Exp. Tech. 55(1), 85-89 (2012).

10. G. M. Mikheev et al., "Spectral dependence of circular photocurrent in silver-palladium resistive films," Tech. Phys. Lett. 40(5), 424-428 (2014).

11. T. Hasan et al., "Nanotube-polymer composites for ultrafast photonics," Adv. Mater. 21(38-39), 3874-3899 (2009).

12. Z. Sun, T. Hasan, and A. C. Ferrari, "Ultrafast lasers mode-locked by nanotubes and graphene," Physica E 44(6), 1082-1091 (2012).

13. M. E. Itkis et al., "Bolometric infrared photoresponse of suspended single-walled carbon nanotube films," Science 312(5772), 413-416 (2006).

14. M. A. Bissett and J. G. Shapter, "Photocurrent response from vertically aligned singlewalled carbon nanotube arrays," J. Phys. Chem. C 114(14), 6778-6783 (2010).

15. S. Cataldo et al., "Carbon nanotubes and organic solar cells," Energy Environ. Sci. 5(3), 5919-5940 (2012).

16. A. Moisala et al., "Single-walled carbon nanotube synthesis using ferrocene and iron pentacarbonyl in a laminar flow reactor," Chem. Eng. Sci. 61(13), 4393-4402 (2006).

17. Y. Tian et al., "Controlled synthesis of single-walled carbon nanotubes in an aerosol reactor," T. Phys. Chem. C 115(15), 7309-7318 (2011).

18. A. Kaskela et al., "Aerosol-synthesized SWCNT networks with tunable conductivity and transparency by a dry transfer technique," Nano Lett. 10(11), 4349-4355 (2010).

19. E. H. Melan, "Stability of palladium oxide resistive glaze films," Microelectron. Reliab. 6, 53-65 (1967).

20. S. F. Wang et al., "Silver-palladium thick-film conductors," J. Am. Ceram. Soc. 77(12), 3051-3072 (1994).

21. G. M. Mikheev, R. G. Zonov, and V. A. Aleksandrov, "Light-induced EMF in silver-palladium film resistors," Tech. Phys. Lett. 36(7), 675-678 (2010).

22. S. F. Wang and W. Huebner, "Thermodynamic modeling of equilibrium subsolidus phase relations in the Ag-Pd-O $\mathrm{O}_{2}$ System," t. Am. Ceram. Soc. 74(6), 1349-1353 (1991).

23. G. M. Mikheev et al., "Effect of the burning temperature on the phase composition, photovoltaic response, and electrical properties of $\mathrm{Ag} / \mathrm{Pd}$ resistive films," Phys. Solid State 56(11), 2286-2293 (2014).

24. V. Deshpande et al., "Properties of lead-free conductive thick films of co-precipitated silverpalladium powders," Mater. Chem. Phys. 93(2-3), 320-324 (2005).

25. D. Olevik et al., "Stability of carbon nanotubes to laser irradiation probed by Raman spectroscopy," Phys. Status Solidi B 245(10), 2212-2215 (2008).

26. G. M. Mikheev et al., "Laser images recording on aerosol synthesized single walled carbon nanotubes films," Tech. Phys. Lett. 41(9), 887-890 (2015).

27. M. S. Dresselhaus, G. Dresselhaus, and A. Jorio, "Raman spectroscopy of carbon nanotubes in 1997 and 2007," J. Phys. Chem. C 111(48), 17887-17893 (2007).

28. Y. Tian et al., "Tailoring the diameter of single-walled carbon nanotubes for optical applications," Nano Res. 4(8), 807-815 (2011). 
29. M. S. Dresselhaus et al., "Raman spectroscopy of carbon nanotubes," Phys. Rep. 409(2), 47-99 (2005).

30. J. R. McBride, K. C. Hass, and W. H. Weber, "Resonance-Raman and lattice-dynamics studies of single-crystal PdO," Phys. Rev. B 44(10), 5016-5028 (1991).

31. W. H. Weber, R. J. Baird, and G. W. Graham, "Raman investigation of palladium oxide, rhodium sesquioxide and palladium rhodium dioxide," T. Raman Spectrosc. 19(4), 239-244 (1988).

32. C. Jiang et al., "Helicity-dependent photocurrents in graphene layers excited by midinfrared radiation of a $\mathrm{CO}_{2}$ laser," Phys. Rev. B 84(12), 125429 (2011).

33. G. M. Mikheev, A. S. Saushin, and V. V. Vanyukov "Helicity-dependent photocurrent in the resistive Ag/Pd films excited by IR laser radiation," Quantum Electron. 45(7), 635-639 (2015).

34. A. S. Saushin, K. G. Mikheev, and G. M. Mikheev, "Application of quarter-wave plate for study the circular photocurrent in nanostructured materials in a wide spectral range," Khim. Fiz. Mezoskopiya 17(2), 305-312 (2015).

Konstantin G. Mikheev is a researcher at the Institute of Mechanics Ural Branch Russian Academy of Sciences. He received his PhD in physics and mathematics in 2013. His research interests include the interaction of laser radiation with carbon nanomaterials and Raman spectroscopy.

Aleksandr S. Saushin graduated in 2012 at Izhevsk State Technical University. Currently, he is a researcher of the laser laboratory of the Institute of Mechanics in Izhevsk. His research interest includes the interaction of laser radiation with matter.

Ruslan G. Zonov is a senior researcher at Institute of Mechanics in Izhevsk. He received his $\mathrm{PhD}$ in physics and mathematics in 2006 . He has over 40 scientific publications and four patents. His research interests associated with the high-power laser radiation interaction with thin metal and carbon films and studying of optoelectronic properties of these films.

Albert G. Nasibulin is a professor at Skolkovo Institute of Science and Technology and an adjunct professor at Aalto University School. He received his PhD in physical chemistry at Kemerovo State University and his Doctor of Science at Saint-Petersburg Technical State University. He has over 180 peer-reviewed scientific publications and 17 patents. He has specialized in the synthesis of nanomaterials, investigation of their growth mechanism, and potential applications in electronics and optoelectronics.

Gennady M. Mikheev graduated in 1981 at Moscow State University, where he also obtained his PhD in 1985. He obtained a DSc in 2000 from the Russian Academy of Science. Currently, he is a professor and the head of the laser laboratory of the Institute of Mechanics in Izhevsk. His research interest includes the interaction of laser radiation with matter. 